phys. stat. sol. (a) 188, No. 1, 411-414 (2001)

\title{
Characterization of 2.5-Inch Diameter Bulk GaN Grown from Melt-Solution
}

V. Soukhoveev ${ }^{1}$ ) (a), V. IVAntsov (a), Yu. Melnik (a), A. Davydov (b),

D. Tsvetkov (a), K. Tsvetkova (a), I. Nikitina (c), A. Zubrilov (c),

A. Lavrentiev (c), and V. DMitriev (a)

(a) TDI, Inc., Gaithersburg, MD 20877, USA

(b) NIST, Gaithersburg, MD, USA

(c) A.F. Ioffe Institute and Crystal Growth Research Center, 194021 St. Petersburg, Russia

(Received July 26, 2001; accepted August 16, 2001)

Subject classification: 61.10.Nz; 68.37.Xy; 78.55.Cr; 81.05.Ea; 81.10.Fq; S7.14

GaN bulk crystals up to 2.5-inch diameter were grown from melt-solution. Properties of these crystals were investigated using Auger electron spectroscopy, X-ray diffraction and photoluminescence.

Introduction Crystal growth from a melt or melt-solution is a widely accepted technique for industrial-scale production of important semiconductor materials. Recently we have demonstrated the growth of 2-inch diameter $\mathrm{GaN}$ crystals by the melt-solution technique [1]. The crystals were grown at about $900{ }^{\circ} \mathrm{C}$ in an ambient pressure not exceeding $2 \mathrm{~atm}$. This paper provides information on the physical properties of these $\mathrm{GaN}$ crystals.

Experimental Growth experiments were conducted in a stainless steel water-cooled chamber with a resistively heated growth zone. The growth zone contained a high purity graphite crucible containing the melt and a set of thermal radiation shields. GaN crystals were grown from Ga-based melt-solution using the procedure described in our prior publications [2]. Growth temperature was around $900{ }^{\circ} \mathrm{C}$ and the pressure in growth chamber during the growth was less than 2 atm. Growth time varied from 5 to $24 \mathrm{~h}$. The growth chamber had several quartz view-ports allowing in-situ observation of the growing crystal.

Auger electron spectroscopy (AES) was used to monitor the nominal composition of grown GaN crystals within an accuracy of 1 at\%. X-ray diffraction (XRD) was employed to evaluate crystal quality of grown GaN material. Photoluminescence (PL) experiments were done at liquid nitrogen temperatures using a $1 \mathrm{~kW}, 10 \mathrm{~ns}$ pulse nitrogen laser emitting at the wavelength of $337.1 \mathrm{~nm}$.

Results GaN crystals up to 2.5-inch diameter were grown (Fig. 1). The thickness of the crystals ranged from 50 to $200 \mu \mathrm{m}$ (Fig. 2a). The AES spectra taken from GaN crystal surface featured gallium and nitrogen related peaks typical for GaN. As expected, the as-grown sample surface contained significant oxygen $\left(\mathrm{O}_{\mathrm{KLL}}, 504 \mathrm{eV}\right)$ and carbon $\left(\mathrm{C}_{\mathrm{KLL}}, 263 \mathrm{eV}\right)$ contamination due to exposure to air. Follow sputtering oxy-

\footnotetext{
1) Corresponding author; Phone: +1 30120883 42; Fax: +1 30133054 00; e-mail: vitali@tdii.com
} 


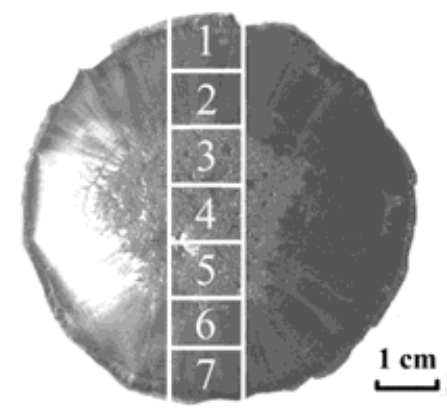

Fig. 1. Photo of GaN crystal grown from liquid phase

gen was not detected in the bulk of the material. In contrast, carbon was observed in all measured AES spectra. Figure 2 shows an image of a cleaved edge of GaN sample obtained by optical microscopy and a transmission electron microscopy (TEM) cross-section image. The TEM image shows that local crystal structure is single crystalline.

In order to obtain information on crystal structure and optical properties of large GaN crystals grown from melt-solutions we investigated in detail the 2.5 -inch GaN crystal shown in Fig. 1. The crystal was diced into seven pieces with a size of $\sim 1 \times 1 \mathrm{~cm}^{2}$. The pieces were numbered as shown in Fig. 1. Each piece was investigated by X-ray diffraction and photoluminescence. In the case of X-ray diffraction measurements the X-ray beam had a size comparable with sample size. For PL study, the diameter of laser beam on sample surface was about $3 \mathrm{~mm}$.

$\mathrm{X}$-ray diffraction measurements were done using both $\omega-2 \theta$ and $\omega$-scanning geometries. Figure 3 demonstrates XRD rocking curves recorded at points 1,3 , and 5 of the $\mathrm{GaN}$ crystal. For $\omega-2 \theta$ scans, the full width at half maximum (FWHM) values of the (0002) reflection were rather small and did not exceed 160 arcsec. For $\omega$-scans, the FWHM values ranged from 13 to 87 arcmin for different pieces. Pieces 3 and 7 had a similar shape of $\omega$-scan rocking curve with a single peak indicating single crystal structure of the material (see Fig. 3b). Pieces 1, 2, 4, 5, and 6 exhibited multi-peak $\omega$-scan rocking curves usual for textured material (Figs. 3a, c).

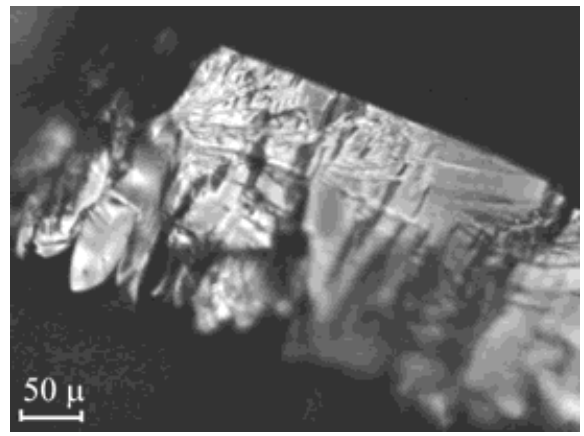

a)

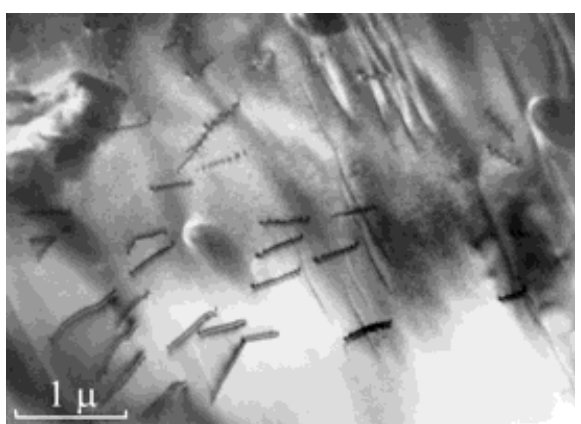

b)

Fig. 2. a) Optical microscope image of cleaved edge and b) TEM cross section image of GaN sample (TEM measurements were done by M. Albrecht, Erlangen University [3]) 
a)

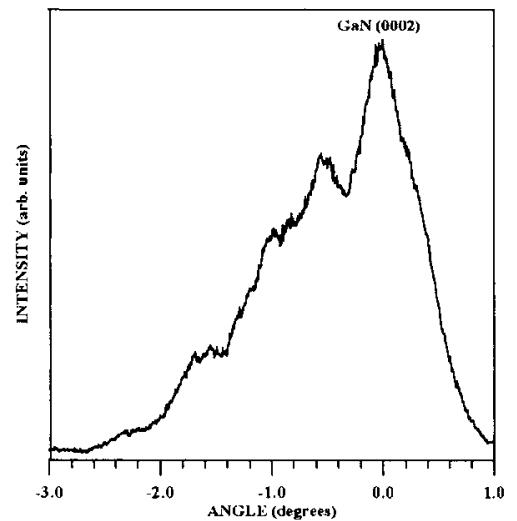

b)

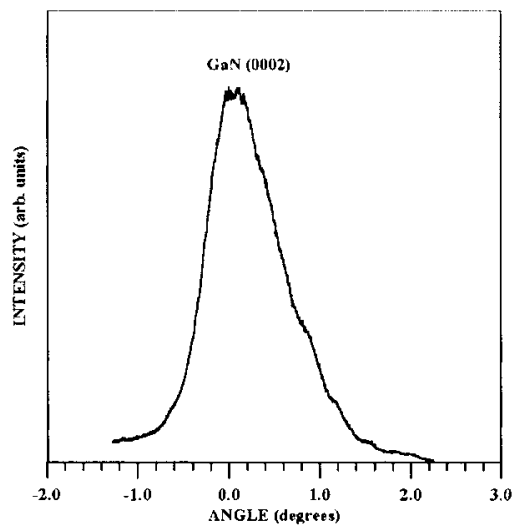

c)

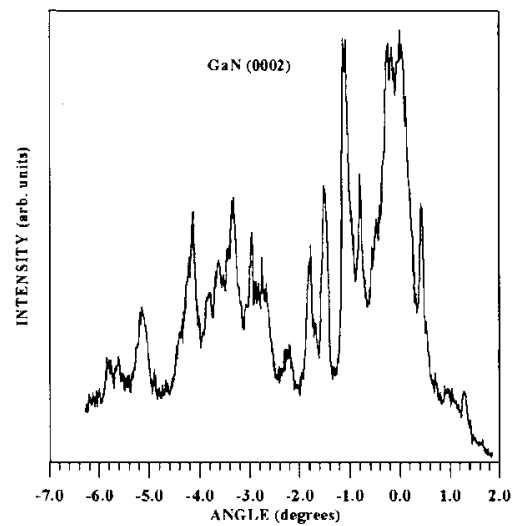

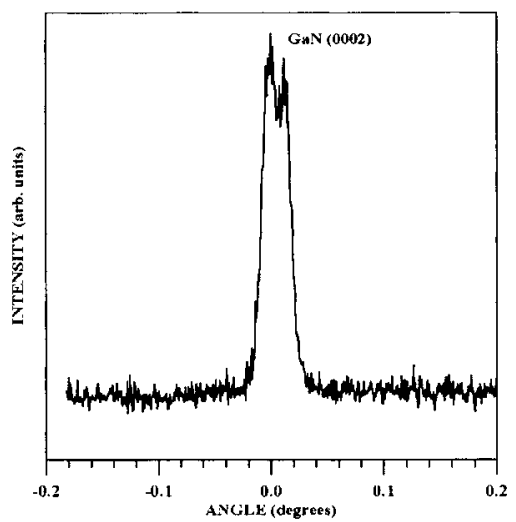
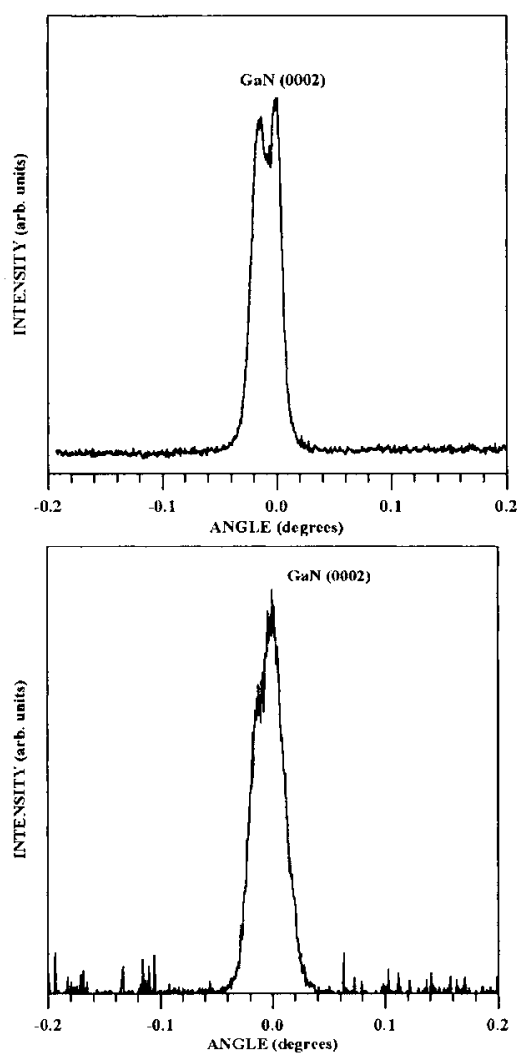

Fig. 3. X-ray rocking curves measured for pieces 1 (a), 3 (b), and 5 (c). X-ray rocking curves measured in $\omega$ - and $\omega-2 \theta$ scanning geometries are shown on the left and right, respectively

The PL spectra of the GaN sample (Fig. 4) contained an edge peak $(\lambda \approx 358 \mathrm{~nm})$ and yellow luminescence peak $(\lambda \approx 550 \mathrm{~nm})$. The spectra measured on different pieces of the sample were similar. The intensities of edge and yellow luminescence were com- 


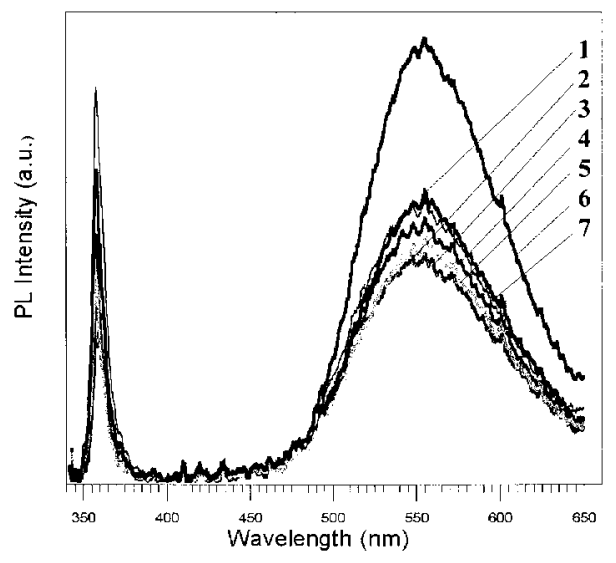

Fig. 4. Photoluminescence spectra measured for different pieces of $\mathrm{GaN}$ sample

parable. The FWHM values for the edge PL peak measured at $77 \mathrm{~K}$ for different pieces of the sample ranged from 53 to $86 \mathrm{meV}$.

Conclusions 2.5-inch diameter $\mathrm{GaN}$ sample was grown from the melt-solution. An $\mathrm{X}$-ray study indicated that the sample consisted of both large grain single crystal regions and highly-textured domains. The FWHM values for $\omega$ - and $\omega-2 \theta$ scan X-ray rocking curves ranged from 13 to 87 arcmin and from 81 to 160 arcsec, respectively. The FWHM of PL edge peak measured for the sample ranged from 53 to $86 \mathrm{meV}$ (77 K).

Acknowledgement This program was supported by ONR (contract monitor Colin Wood).

\section{References}

[1] V. Soukhoveev et al., International Specialist Meeting on Bulk Nitride Growth and Related Techniques, November 2000, Brazil.

[2] V. A. SukhoveYev et al., Mater. Res. Soc. Symp. Proc. 595, W6.6.1 (1999).

[3] M. AlbReCht et al., "Structure and optical properties of GaN crystals grown from the liquid phase", Fall MRS 2000, Boston. 